\title{
Application of Three-Dimensional Magnetic Resonance Imaging in the Diagnosis of Perianal Abscess
}

\author{
Fang Zhang1, Shan Xiong ${ }^{1}$, Sibin Liu ${ }^{1}$, Peng Xia ${ }^{2 *}$ \\ ${ }^{1}$ Department of Radiology, Jingzhou Central Hospital, Jingzhou, China \\ ${ }^{2}$ Medical School of Yangtze University, Jingzhou, China \\ Email: *372856250@qq.com
}

How to cite this paper: Zhang, F., Xiong, S., Liu, S.B. and Xia, P. (2019) Application of Three-Dimensional Magnetic Resonance Imaging in the Diagnosis of Perianal Abscess. Health, 11, 535-545. https://doi.org/10.4236/health.2019.115046

Received: April 15, 2019

Accepted: May 24, 2019

Published: May 27, 2019

Copyright $\odot 2019$ by author(s) and Scientific Research Publishing Inc. This work is licensed under the Creative Commons Attribution International License (CC BY 4.0).

http://creativecommons.org/licenses/by/4.0/

\section{(c) () Open Access}

\begin{abstract}
Perianal abscess is a common disease in anorectal surgery. If the diagnosis is not clear and the cure is thoroughly cleared, the recurrence and spread of anal fistula will cause life-long pain. Objective: To investigate the application of 3.0T MRI 3D CUBE T2WI lipid suppression sequence in the diagnosis of perianal abscess. Methods: Thirty-six patients with perianal abscess confirmed by operation were examined with 2D T2WI and 3D CUBE T2WI lipid suppression sequences before operation. Two imaging techniques were evaluated to show the types of perianal abscess, the number of abscesses, the number of internal orifices of abscess, and the number of fistula branches with anal fistula in abscess. Results: Among 36 cases of perianal abscess, there were 5 cases of anal subcutaneous abscess, 12 cases of ischiorectal space abscess ( 8 cases complicated with anal fistula), 6 cases of posterior anal space abscess, 5 cases of anal sphincter abscess ( 3 cases complicated with anal fistula), 2 cases of high intermuscular abscess, 2 cases of rectal submucosal abscess, 3 cases of complex abscess ( 3 cases complicated with anal fistula), 1 case of misdiagnosis, 2D T2WI lipid suppression sequence and 3D CUBE T2WI suppression. The accuracy of lipid sequence abscess typing was $80.6 \%$ (29/36) and $88.9 \%$ (32/36), respectively, with no significant difference $(\mathrm{P}>0.05)$. Thirty-six patients were surgically diagnosed as having 32 internal orifices, $68.8 \%(22 / 32)$ and $93.8 \%(30 / 32)$ of $2 \mathrm{D}$ T2WI and 3D CUBE T2WI lipid-suppressing sequences, respectively, with significant difference $(\mathrm{P}<0.05)$; among the 36 patients, 14 had perianal abscess complicated with anal fistula, and 21 fistulas were found during the operation, 21 cases were found fistula, $61.9 \%(13 / 21)$ of fistula branches were found by 2D T2WI lipid-suppressing sequence and 90.5\% (19/21) of 3D CUBE T2WI lipid-suppressing sequence. The difference was statistically significant $(\mathrm{P}<0.05)$. The number of perianal abscesses was
\end{abstract}


identical between the two scanning techniques. Conclusion: 3D CUBE T2WI lipid suppression sequence is superior to 2D T2WI lipid suppression sequence in the classification of perianal abscess, the number of internal orifices of abscess and the number of fistula branches of abscess complicated with anal fistula. It can also determine the number of internal orifices of abscess complicated with anal fistula, the number of fistula branches, the shape of primary and branch fistula and the relationship among pelvic floor muscle tissues. It can provide more accurate images for preoperative and intraoperative clinical surgery.

\section{Keywords}

Magnetic Resonance Imaging, Three-Dimensional Imaging, Perianal Abscess

\section{Introduction}

Perianal abscess is a common disease in anorectal surgery. In the early stage, due to inflammation, injury, surgery and other reasons, the glands around the anus are blocked, which causes a large number of bacteria to multiply and gradually form perianal abscess. In the later stage, the abscess spreads to the periphery and even ruptures to form an anal fistula [1]. Finally, it involves different perianal spaces and develops into different types of perianal abscess and anal fistula. With good soft tissue resolution, MRI has become the gold standard for the evaluation and classification of perianal diseases in developed countries [2]. Recently, ultrasound and transanal ultrasound have been widely used in the diagnosis of perianal abscess. However, ultrasonography is valuable in the diagnosis of perianal abscess, but MRI can show the lesions directly and clearly. The relationship between perianal abscess and surrounding tissues and the fistula course of perianal abscess complicated with anal fistula can also be shown more accurately. Zhang Shanhong et al. found that the correct rate of diagnosing perianal abscess with different MRI sequences is significantly higher than that with ultrasound, and has statistical significance [3]. Cheng Jing et al. showed that high field intensity MRI could accurately display the location of perianal abscess, clearly show the relationship between anal fistula and perianal tissue, and could achieve typing diagnosis [4]. Those two researches both used 1.5T MRI scanner and conventional imaging sequence; our study used 3.0T high field intensity MRI 3D CUBE three-dimensional imaging technology, whether enhanced or plain scan will greatly improve the diagnosis of perianal abscess and anal fistula compared with the previous traditional technology. 3D CUBE is a new technique for three-dimensional imaging scanning of MRI. It is an isotropic three-dimensional fast echo pulse sequence. Its scanning time is short and its plane is thin. It can rotate images arbitrarily in different directions and angles. Its sensitivity and accuracy are higher than those of the two-dimensional sequence of MRI [5]. In 
this paper, the application value of 3D MRI in perianal abscess was analyzed.

\section{Materials and Methods}

\subsection{Clinical Data}

36 patients with perianal abscess diagnosed by transonic surgery from August 2016 to August 2018 were collected randomly from Jingzhou central hospital. Among them, there were 28 males and 8 females, aged 23-64 years, with an average age of about 37 years. All patients had a history of perianal swelling and pain, and some had fever. Four of them had a history of perianal abscess surgery and one had a history of anal foreign body removal. All cases were confirmed by surgery.

\subsection{MRI Test}

GE Signa 3.0T (GE Healthcare, Milwaukee, Wisconsin, USA) magnetic resonance scanner was used with phased array coils. No medicine and intestinal preparation, no markers and no clinical intervention were needed. First, pelvic sagittal scanning was performed to find the median section of the anal canal, and as the reference plane, the plane perpendicular to the anal canal was the axis, and the plane parallel to the anal canal was the coronal position; the three directions were observed in sagittal, axial and coronal positions. Conventional 2D scanning sequence: FSE T1WI axis, T2W (sagittal, axial, coronal) lipid suppression sequence, DWI; scanning layer thickness $6 \mathrm{~mm}$, layer spacing $1 \mathrm{~mm}$. 3D imaging sequence: FSE axis 3D CUBE T2WI lipid suppression sequence, $1.5 \mathrm{~mm}$ thickness, $0 \mathrm{~mm}$ interval, 3-D image to ADW4.6 workstation for post-processing.

\subsection{Data Analysis}

Two senior doctors who have been engaged in abdominal and pelvic imaging diagnosis for many years read and analyzed the films together. When the diagnosis is inconsistent, the diagnosis is confirmed by comprehensive analysis. The classification of perianal abscess, the number of internal orifices of abscess and the number of fistula branches in abscess were mainly evaluated. All cases were diagnosed and treated by operation and pathology, and compared with the diagnosis of MRI. The specific situation of perianal abscess and anal fistula complicated by abscess was recorded.

\subsection{Statistical Methods}

Statistical software SPSS18.0 was used to analyze and process the data. The lipid suppression sequences of $2 \mathrm{D}$ T2WI and 3D CUBE T2WI were compared. The percentage of all counting data was used and $\mathrm{X} 2$ test was used. The difference was statistically significant with $\mathrm{P}<0.05$.

\section{Results}

\subsection{Clinical Characteristics of Perianal Abscess Cases}

Of the 36 patients, 28 were males and 8 were females, aged from 23 to 64 years, 
with an average age of about 37 years. All patients had different degrees of anal swelling, perianal swelling and pain history. Some patients touched the mass and had fever. Four patients had a history of surgical treatment of perianal abscess and one had a history of resection of foreign body in the anus. All cases were confirmed by surgery. Among 36 cases of perianal abscess, there were 5 cases of anal subcutaneous abscess, 12 cases of sciatic-rectal space abscess ( 8 cases complicated with anal fistula), 6 cases of posterior anal space abscess, 5 cases of anal sphincter abscess ( 3 cases complicated with anal fistula), 2 cases of high intramuscular abscess, 2 cases of rectal submucosal abscess, 3 cases of complex abscess ( 3 cases complicated with anal fistula), 1 case of misdiagnosis, and 1 case of postrectalteratoma confirmed by operation (Table 1).

\subsection{D CUBE T2WI Lipid Suppression Sequence Is Significantly Better than 2D T2WI Lipid Suppression Sequence during Diagnosis of Perianal Abscess}

MRI 2D T2WI and 3D CUBE T2WI lipid suppression sequences were consistent with surgical results in showing the number of perianal abscesses. 3D CUBE T2WI lipid suppression sequence was superior to 2D T2WI lipid suppression sequence in the number of perianal abscess internal orifices and the number of anal fistula branches associated with abscess (Table 2). Figure 1(a) sagittal T2WI lipid suppression sequence showed irregular patchy high signal in front of rectum, but it was not clear whether the lesion was directly related to rectum and whether it involved rectum. Figure 1(b) sagittal 3D CUBE T2WI lipid suppression sequence could clearly show irregular patchy high signal in front of rectum and submucosa. The boundary of abscess lesion was very clear, and the lesion involved straight. Although there was no significant difference between the two techniques in the classification of perianal abscess, the accuracy and localization of 3D CUBE T2WI lipid suppression sequence was better than that of 2D T2WI

Table 1. Clinical Classification and characteristics of 36 cases of perianal abscess.

\begin{tabular}{|c|c|c|}
\hline Clinical classification & $\mathrm{n}$ & Clinical features \\
\hline Perianal subcutaneous abscess & 5 & $\begin{array}{l}\text { Palpation with lumps } \\
\text { and mild pain }\end{array}$ \\
\hline Abscess of ischiorectal space & $\begin{array}{c}12 \text { (8 cases complicated } \\
\text { with anal fistula) }\end{array}$ & Anal flatulence discomfort \\
\hline Abscess of posterior anal space & 6 & Redness and swelling of skin \\
\hline Abscess of anal sphincter space & $\begin{array}{c}5 \text { (3 cases complicated with } \\
\text { anal fistula) }\end{array}$ & $\begin{array}{l}\text { Pain and discomfort, } \\
\text { partial late burst }\end{array}$ \\
\hline Submucosal abscess of rectum & 2 & $\begin{array}{l}\text { Anal swelling pain, } \\
\text { finger tenderness }\end{array}$ \\
\hline High intermuscular abscess & 2 & $\begin{array}{l}\text { Hidden onset, tenderness } \\
\text { of the mass }\end{array}$ \\
\hline Complex abscess & $\begin{array}{c}3 \text { (3 cases complicated with } \\
\text { anal fistula) }\end{array}$ & $\begin{array}{l}\text { Swollen, hot and painful, } \\
\text { touching lumps }\end{array}$ \\
\hline $\begin{array}{c}\text { Misdiagnosis } \\
\text { (Posterior rectal teratoma) }\end{array}$ & 1 & Touching the block \\
\hline
\end{tabular}


Table 2. Comparison of the results of perianal abscess visualization and operation by two kinds of MRI in 36 patients [n(\%)].

\begin{tabular}{ccccc}
\hline Operative resultn & 2D & 3D & $\chi^{2}$ & P \\
\hline $\begin{array}{c}\text { Classification of } \\
\text { perianal abscess 36 }\end{array}$ & $29(80.6)$ & $32(88.9)$ & 0.966 & 0.326 \\
$\begin{array}{c}\text { Internal orifice of } \\
\text { perianal abscess 32 }\end{array}$ & $22(68.8)$ & $30(93.8)$ & 6.564 & 0.01 \\
$\begin{array}{c}14 \text { cases of perianal } \\
\text { abscess complicated } \\
\text { Fistula count 21 }\end{array}$ & $13(61.9)$ & $19(90.5)$ & 4.725 & 0.03 \\
Perianal abscess 38 & $38(100)$ & $30(100)$ & & \\
\hline
\end{tabular}

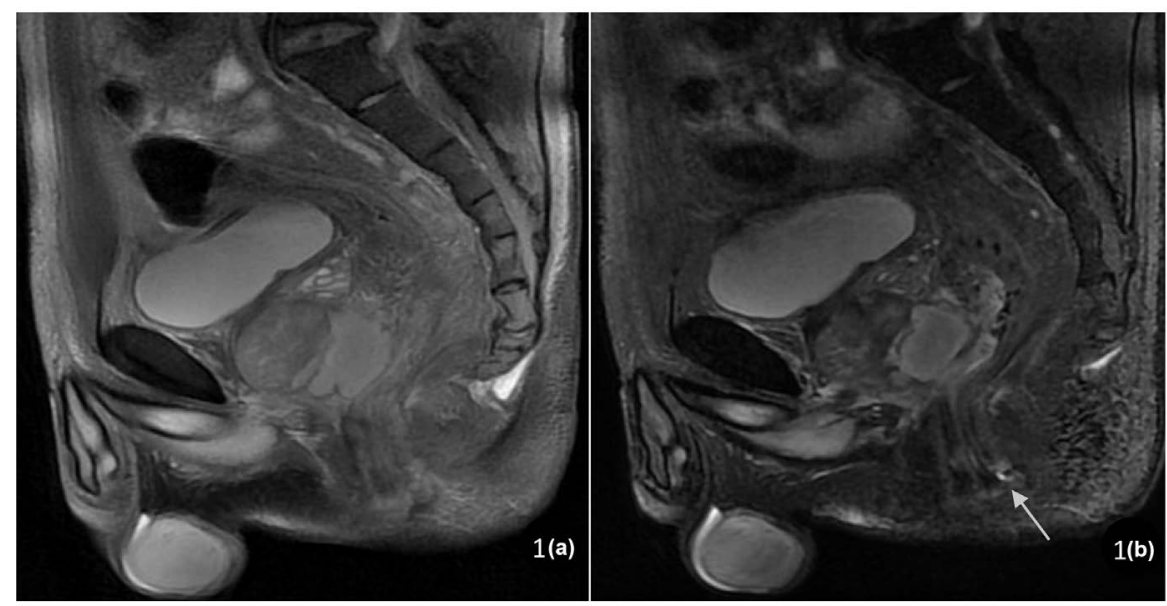

Figure 1. (a) Sagittal T2WI lipid suppression sequence showed irregular patchy high signal in front of rectum, unclear boundary and close to rectum, and no obvious abnormal signal in back of anus; (b) Sagittal 3D CUBE T2WI lipid suppression sequence showed irregular patchy high signal in front of rectum and submucosa, with clear boundary and strip high signal in submucosa of rectum, and a small abscess shadow (fine arrow) in back of anus proved to be submucosal abscess of rectum after operation.

lipid suppression sequence on imaging images. 3D CUBE T2WI lipid suppression sequence is superior to $2 \mathrm{D}$ T2WI lipid suppression sequence in showing the number of internal orifices of perianal abscess and the number of fistula branches of abscess complicated with anal fistula. Figure 2(a) axial T2WI lipid suppression sequence showed a small strip high signal on the left side of the anus and no obvious abnormal duct-like signal around it. Figure 2(b) axial 3D CUBE T2WI lipid suppression sequence showed a small strip high signal on the left side of the anus with clear boundary. Two small duct-like high signal fistulas could be seen in the middle and lower part of the lesion. The number and course of branches of the anal fistula accompanied by abscess were shown. Indeed, highlighting the advantages of 3D imaging technology in precision and accuracy, surgical confirmation of sphincter abscess complicated with anal fistula was made. Figure 3(a) coronal T2WI lipid suppression sequence showed irregular patchy high signal in the left rear of the anus and small strip high signal above 

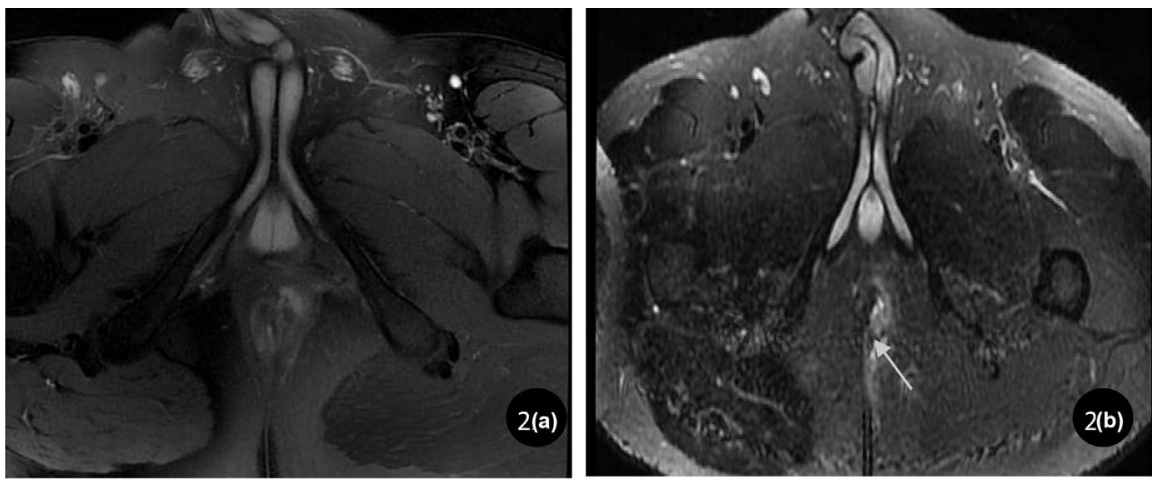

Figure 2. (a) T2WI lipid suppression sequence showed a strip of high signal on the left side of the anus and no abnormal duct-like signal around it.; (b) Axial 3D CUBE T2WI lipid suppression sequence showed a strip-like high signal on the left side of the anus with clear margins. Two small canalicular structures with high signal-fistula shadows (arrows) could be seen in the middle and lower part of the lesion. Surgical confirmation of sphincter abscess complicated with anal fistula was made.

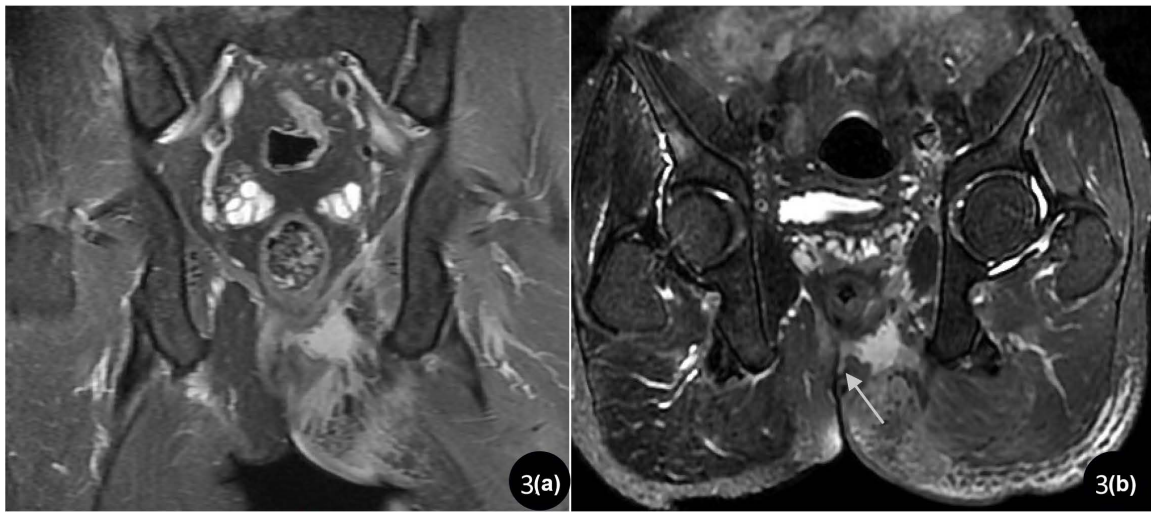

Figure 3. (a) Coronal T2WI lipid suppression sequence showed irregular patchy high signal in the left rear of the anus and small strip high signal above the anal sphincter at about six points.; (b) Coronal 3D CUBE T2WI lipid suppression sequence clearly showed that a high signal like a conduit was connected with a high signal like a patch of adjacent mass in the direction of 6 o'clock in the anus and crossed the external anal sphincter. Surgical confirmation was that abscess of ischiorectal space with anal fistula.

the anal sphincter at about 6 o'clock, while Figure 3(b) coronal 3D CUBE T2WI lipid suppression sequence clearly showed that there was a high signal like a pipeline connected with the adjacent lesion at 6 o'clock and crossed the external anal sphincter, which was confirmed by surgery as perianal abscess with anal sphincter fistula. When comparing the two techniques, three-dimensional imaging was more sensitive and accurate in detecting and displaying lesions in axial, sagittal and coronal positions, especially for perianal abscess complicated with anal fistula. Figure 4(a) sagittal T2WI lipid suppression sequence showed a small strip of high signal behind the anus, which could only display part of the lesion length. Figure 4(b) sagittal 3D CUBE T2WI lipid suppression sequence could display the full length of the lesion behind the anus, which was twice the length of the lesion displayed by $2 \mathrm{D}$ sequence. It fully demonstrated the advantages of three-dimensional imaging tech- 
nology in the integrity and continuity of the diagnosis of the lesion. (Figure 4(c), Figure 4(d)) Three-dimensional VR imaging images of 3D CUBE can be used to observe the lesions from different directions. After image processing, the lesions of perianal abscess can be displayed more clearly and visually.

\section{Discussion}

Perianal abscess is a common surgical disease in clinic. It usually spreads from anal infection to anal canal. As for perirectal space formation, the incidence of anorectal diseases accounted for $25 \%$. Perianal abscess is more common in both adults and infants than in females. The sex difference of infants is more obvious, and the ratio of males to females can reach 9:1. Recent studies have shown that the liquid and cellular immune functions of men are lower than those of women, and men are more susceptible to most infectious diseases than women. Infection is the main cause of the disease. Pathology can be divided into early stage (inflammatory infiltration stage), middle stage (pyogenic stage) and late stage (ulceration stage). When the pus gathers and drains poorly, it can cause pain. Most patients can touch the tumors around the anus. If the pus stimulates after bursting, it can cause itching of the skin around the anus. Initial patients may have
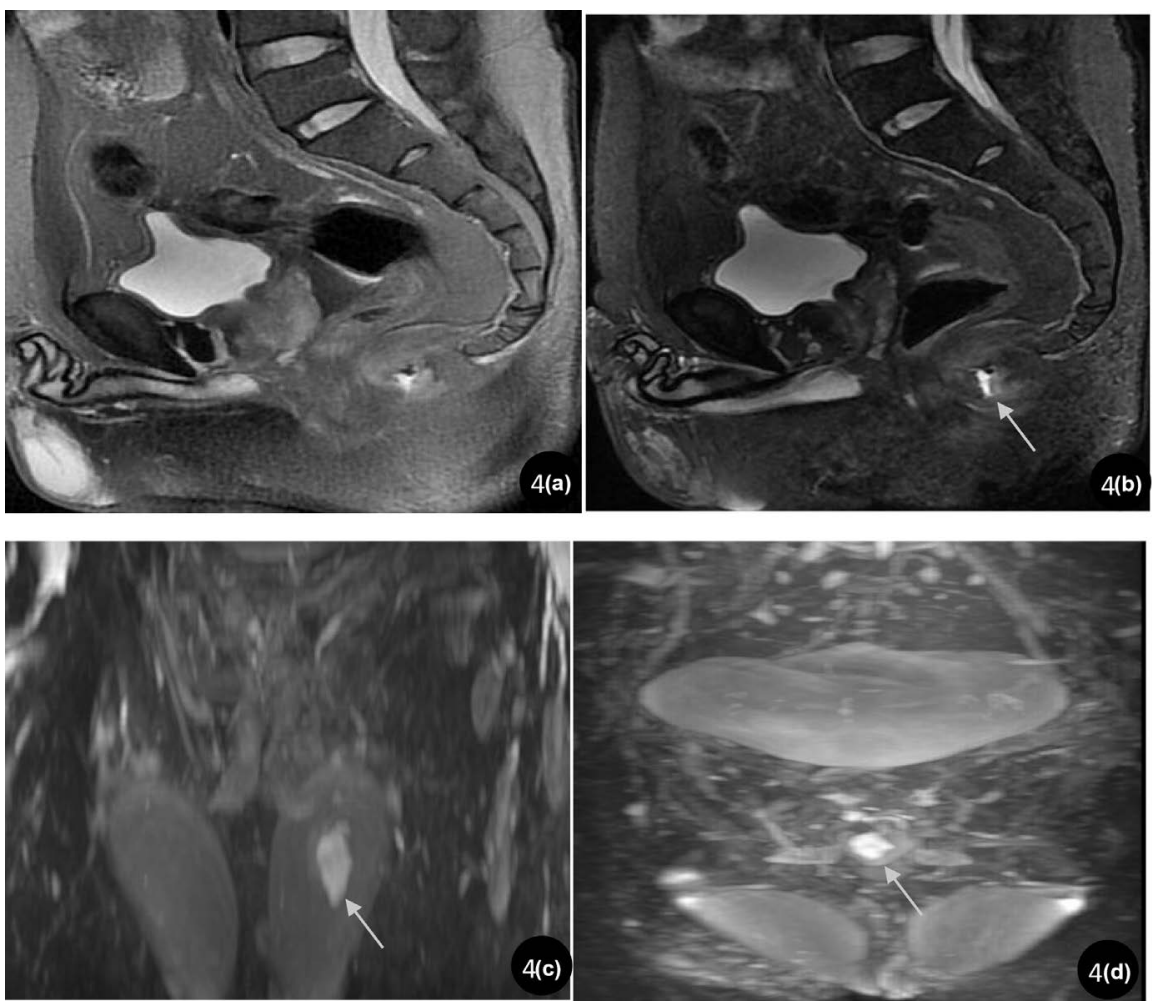

Figure 4. (a) Sagittal T2WI lipid suppression sequence showed a small strip of high signal behind the anus, which could only show about $6 \mathrm{~mm}$ in length.; (b) The sagittal 3D CUBE T2WI lipid suppression sequence showed a strip-like high signal in the back of the anus, which could show the whole length of about $12 \mathrm{~mm}$ (thin arrow). Surgical diagnosis was abscess of posterior anal space; (c)-(d) Axis and coronal 3D post-processing image VR can clearly and stereoscopically display high signal of pus cavity (arrow). 
no systemic symptoms, such as fever and other systemic symptoms if the scope of aggravation of infection is expanded. Severe pain can cause difficulty in urinating and defecating. Clinical classification, according to the location of abscess, the abscess above levatorani muscle (high abscess) is divided into: pelvic and rectal space abscess, rectal submucosal abscess, posterior rectal space abscess and high horseshoe abscess; the abscess below levatorani muscle (low abscess) is divided into: ischiorectal space abscess, perianal subcutaneous abscess, posterior anal space abscess and low horseshoe abscess.

Surgery is the best treatment for perianal abscess. When abscess is incised, the scope of the incision of anal sphincter is related to the internal orifice. Failure to thoroughly clean the residual internal orifice is an important factor causing recurrence. Therefore, clearing the location of the internal orifice is important information that must be known before operation. According to the European and American guidelines, when the position of the abscess's internal orifice is low and the internal orifice can be treated at the same time as drainage to avoid the recurrence of anal fistula in the later stage [6]. Before the operation, the classification of perianal abscess, the number, scope, overall shape, the internal orifice of abscess, the number of branches with or without anal fistula and fistula were defined. The relationship between perianal abscess, sphincter and pelvic floor muscle tissue could be established as early as possible, and effective and accurate surgical methods and treatment programs could be worked out, to minimize the impairment of anorectal function.

Many literatures have confirmed the importance of MRI in the diagnosis of perianal abscess. Jose C. et al. showed that the new MR diffusion and perfusion sequences could allow clinical surgeons to evaluate the perianal manifestations of Crohn's disease with different anal fistula canals [7]. The imaging information provided could quantify the severity of the disease and evaluate the clinical response. 2D T2WI uses chemical shift effects of water and fat to inhibit high-signal adipose tissue. Other tissue signals have less influence on the sequence, so it can clearly show the anatomical structure of various tissues. $2 \mathrm{D}$ T2WI lipid suppression sequence can clearly show the lesion itself and surrounding tissues in a single abscess, however, the shape of the lesion showing abscess is incomplete. In this case, the length of the lesion displayed by $2 \mathrm{D}$ sequence is half of that of 3D sequence. Especially in the condition of sufficient abscess, the classification of abscess and anal fistula is inaccurate in complex abscess and complicated anal fistula. Especially in the case of fewer abscesses, the residual fibrous scar is not easy to diagnose, and the location of the inner mouth is more uncertain. Therefore, the diagnosis of perianal abscess by MRI 2D T2WI lipid suppression sequence has certain limitations. In China, the imaging examination of perianal abscess is mainly plain magnetic resonance scanning, but in recent years, the reports of enhanced MRI scanning have gradually increased. The axial T1WI lipid suppression enhanced scan can better show the internal and external sphincters. This method can clearly show the lower position of ab- 
scess, such as the abscess among sphincters and the abscess among the sciatic and anal canals and so on.

3D CUBE is a kind of three-dimensional imaging technology [8]. It uses super-long echo chain and variable flip angle technology to acquire 3D FSE sequence in a large area, so that the echo can reach a relatively stable state. It can overcome the limitation of single tissue imaging in one scan of MRI, and ensure the clarity, contrast and signal-to-noise ratio of the image. The signal differences of the perianal anatomical structures on the 3D CUBE sequence are more clearly displayed. The thin 3D CUBE sequence can clearly and accurately display the overall anatomical morphology of perianal abscess, clearly classify it, and observe the concealed inner orifice and deep small abscess cavity. In this study, thin-layer scanning could clearly show irregular patchy high signal in the anterior rectum and submucosa. The boundary of abscess lesion was very clear. The lesion involved the submucosa of rectum. A small abscess shadow was also found behind the anus. The classification of submucosal abscess of rectum was clear. The deep small abscess which was difficult to find or couldn't be clearly displayed by 2D sequence was also observed. Complex abscess complicated with anal fistula has more overall shape, internal and external orifices and fistula branches, and the direction of the fistula is complex. It is easy to make diagnostic errors. The 3D CUBE sequence is particularly prominent in the overall clarity and continuity of the abscess complicated with fistula. The length and direction of main fistula and branch fistula, the relationship between the main fistula and the surrounding tissues and the anal sphincter can be clearly displayed, which is more accurate than $2 \mathrm{D}$ sequence. The $3 \mathrm{D}$ CUBE sequence can reduce the probability of missed diagnosis and misdiagnosis. When the pus is large and sufficient, the overall clarity of the lesion will be greatly improved; but the pus is less or none. Because of the high sensitivity and precision of three-dimensional imaging technology, the main fistula and branch fistula can also be correctly distinguished. The less pus is mixed sign, no pus, the lower signal of the fibrous tubewall. Although the scanning time of 3D CUBE sequence is slightly longer, the volume image obtained by scanning can be observed and reconstructed from any orientation; without scanning axis, vector and crown position separately, only one orientation can be scanned, and the other two orientations can be reconstructed, thus saving the scanning time of two orientations, so the total inspection time is reduced.

The 3D CUBE sequence was applicated in domestic and foreign countries; Patzig $\mathrm{M}$ found that the number of lesions found by using conventional $2 \mathrm{D}$ sequence in multiple sclerosis of the brain was significantly lower than that by using 3D CUBE sequence [9]. Lefevre N. et al. reported that the specificity and sensitivity of 3D CUBE sequence on partial tear of anterior cruciate ligament of knee joint was significantly better than that of conventional 2D MRI sequence [10]. However, there are few reports on the study of perianal abscess and anal fistula by 3D CUBE T2WI lipid suppression sequence. This study shows that 3D 
CUBE T2WI lipid suppression sequence is superior to 2D T2WI lipid suppression sequence in the diagnosis of perianal abscess in showing the overall shape, classification, internal orifice and abscess complicated with anal fistula. The authors believe that it is closely related to the accuracy, sensitivity and stereoscopic effect of 3D CUBE sequence in displaying lesions.

Many literatures have reported the application value of different sequences of MRI in perianal abscess. The 3D CUBE T2WI lipid suppression sequence applied in this study has not been compared with other sequences, and needs to be further improved in future research.

To sum up, 3.0T MRI 3D CUBE T2WI lipid suppression sequence is more clear, intuitive and accurate than conventional 2D T2WI lipid suppression sequence in showing the overall shape of perianal abscess, abscess classification, internal orifice and the number of fistula branches of abscess complicated with anal fistula. It can be used as a routine examination method. It provides accurate opinions and imaging information for the selection of surgical methods of anorectal surgeons, and is worthy of clinical application.

\section{Conflicts of Interest}

The authors declare no conflicts of interest regarding the publication of this paper.

\section{References}

[1] Wang, W.Z., Zhang, Y. and Wang, Z. (2007) Magnetic Resonance Imaging of Anal Fistula. International Medical Clinical Radiology Register, 30, 358-360.

[2] Van Koperen, P.J., Horsthuis, K., Bemelman, W.A, et al. (2008) Perianal Fistulas: Developments in the Classification and Diagnostic Techniques, and a New Treatment Strategy. The Nederlands Tijdschrift voor Geneeskunde, 152, 2774-2780.

[3] Zhang, S.H., Wang, X.L., Liang, X.S., et al. (2017) The Application of Different Imaging Sequences of MRI in the Diagnosis and Classification of Perianal Abscess. Chinese and Foreign Medical Treatment, 32,176-177,180.

[4] Cheng, J., Yun, C., Zhao, X. and Liu, W. (2017) MRI Diagnosis of Perianal Abscess and Anal Fistula. Journal of Medical Imaging, 27, 302-305.

[5] Ai, T., Zhang, W., Priddy, N.K. and Li, X. (2012) Diagnostic Performance of CUBE MRI Sequences of the Knee Compared with Conventional MRI. Clinical Radiology, 67, 58-63. https://doi.org/10.1016/j.crad.2012.07.020

[6] Shi, R.J. (2018) Review of New Progress in Diagnosis and Treatment of Perianal Abscess. Chinese Journal of Integrated Traditional Chinese and Western Medicine, 38, 399-401.

[7] Gallego, J.C. and Echarri, A. (2018) Role of Magnetic Resonance Imaging in the Management of Perianal Crohn's Disease. Insights into Imaging, 9, 47-58. https://doi.org/10.1007/s13244-017-0579-9

[8] Wan, C.H., Zheng, G., Hu, J.W., et al. (2011) Application Value of 3.0T Magnetic Resonance Whole Body Nerve Root Imaging in Neurobrachialvenomatosis. Radiologic Practice, 26, 988-991.

[9] Patzig, M., Burke, M., Brückmann, H. and Fesl, G. (2014) Comparison of 3D Cube 
FLAIR with 2D FLAIR for Multiple Sclerosis Imaging at 3 Tesla. Fortschr Röntgenstr, 186, 484-488. https://doi.org/10.1055/s-0033-1355896

[10] Lefevre, N., Naouri, J.F., Bohu, Y., Klouche, S. and Herman, S. (2014) Partial Tears of the Anterior Cruciate Ligament: Diagnostic Performance of Isotropic Three-Dimensional Fast Spin Echo (3D-FSE-Cube) MRI. European Journal of Orthopaedic Surgery \& Traumatology, 24, 85-91.

https://doi.org/10.1007/s00590-012-1135-4 